\title{
Uso de la Realidad Aumentada como Recurso Didáctico en la Enseñanza Universitaria
}

\author{
Julio Cabero-Almenara(1), Esteban Vázquez-Cano ${ }^{(2)^{\star}}$ y Eloy López-Meneses ${ }^{(3)}$ \\ (1) Universidad de Sevilla, Facultad de Ciencias de la Educación. Calle Pirotecnia, s/n. 41013, \\ Sevilla-España (e-mail: cabero@us.es) \\ (2) Universidad Nacional de Educación a Distancia (UNED), Facultad de Educación Ciencias Sociales. \\ Calle Juan del Rosal, 14. 28040, Madrid-España (e-mail: evazquez@edu.uned.es) \\ (3) Universidad Pablo de Olavide, Facultad de Ciencias Sociales. Carretera de Utrera, km. 1. 41013, \\ Sevilla-España (e-mail: elopmen@upo.es)
}

*Autor a quien debe ser dirigida la correspondencia

Recibido May. 29, 2017; Aceptado Jul. 25, 2017; Versión final Ago. 24, 2017, Publicado Feb. 2018

\begin{abstract}
Resumen
La presente investigación se enmarca dentro del proyecto de investigación español (I+D+I) Realidad aumentada para aumentar la formación. Diseño, producción y evaluación de programas de realidad aumentada para la formación universitaria" (EDU-5746-P-Proyecto RAFODIUN). Se analiza una experiencia de innovación universitaria con Realidad Aumentada con 117 estudiantes que cursan asignaturas de Tecnología de la Información y la Comunicación en la Universidad Pablo de Olavide de Sevilla (España). Se ha aplicado una metodología de investigación cualitativa mediante el diseño de un cuestionario abierto que permite analizar con mayor profundidad la funcionalidad, limitaciones y posibilidades formativas de la realidad aumentada por estudiantes que han empleado la misma en sus procesos formativos. El análisis se ha realizado con ayuda del programa Atlas-Ti y los resultados muestran que la realidad aumentada es una tecnología emergente con amplias posibilidades formativas. Sin embargo, se precisa de una formación e inversión económica para garantizar su éxito en las aulas.
\end{abstract}

Palabras clave: educación tecnológica; realidad aumentada; recursos didácticos; innovación universitaria; educación superior.

\section{Use of Augmented Reality Technology as a Didactic Resource in University Teaching}

\begin{abstract}
The present research is part of the Spanish research project $(I+D+I)$ Augmented reality to foster training. Design, production and assessment of augmented reality programs for University education (EDU-5746-PProject RAFODIUN). An experience of University innovation with Augmented Reality is analyzed with the participation of 117 students who study subjects of Information and Communication Technology at Pablo de Olavide University in Seville (Spain). A qualitative research methodology has been applied through the design of an open questionnaire that allows to deeper analyze the functionality, limitations and formative possibilities of augmented reality by students who have used it in their formative processes. The analysis was carried out with the help of the Atlas-Ti program and the results show that augmented reality is an emerging technology with broad training possibilities. However, it requires training and economic investment to guarantee its success in the classroom.
\end{abstract}

Keywords: technological education; augmented reality; didactic resources; university innovation; higher education. 


\section{INTRODUCCIÓN}

La integración de las Tecnologías de la Información y Comunicación (TIC) en la sociedad, ha venido siendo una de las líneas de investigación más recurrentes en las últimas dos décadas, por citar algunos aportes del estado que guardan relación con la ubicuidad y movilidad, sobresalen (Furth, 2011; Villalustre, 2016). En los últimos 15 años los sistemas educativos universitarios han propiciado una serie de alternativas y propuestas para la educación utilizando Internet como medio de comunicación y tratando de desplazar en un alto porcentaje las clases presenciales (Sevillano y Vázquez-Cano, 2015). Asimismo, el desafío para las universidades, en este marco, reside en rediseñar sus matrices formativas alrededor de las competencias profesionales más que alrededor de las tradicionales asignaturas de forma que se potencie el desarrollo de propuestas didácticas que involucren el trabajo colaborativo para el fomento de un aprendizaje significativo y el incremento progresivo en la actividad docente de la utilización de los recursos educativos electrónicos que combinen diferentes aproximaciones más creativas y colaborativas (Bressler y Bodzin, 2013; VázquezCano et al., 2015; Álvarez-Marín et al., 2017).

En este sentido, la realidad educativa y tecnológica en las aulas de los diferentes niveles académicos, en nuestro sistema educativo en la actualidad, viene de la mano de la incorporación de nuevas herramientas que acerquen a los alumnos y alumnas, de forma sencilla, lúdica y formativa, a los contenidos curriculares. Una de las tecnologías que toman mayor impulso e importancia en la actualidad es la Realidad Aumentada, la cual se ha ido abriendo camino, especialmente en la educación superior (Chang, et al., 2013; Cuendet, et al., 2013; Cabero y García, 2016; Barroso y Gallego-Pérez, 2017). Hoy en día, en los escenarios formativos cada vez están teniendo más auge tecnologías emergentes como la Realidad Aumentada (RA en adelante) (Kipper y Rampolla, 2012; Moreno-Martínez y Leiva-Olivencia, 2017); esta tecnología se estima que tiene un horizonte de implantación en los centros educativos de 3 a 5 años (Johnson et al., 2013). También, se expresa en el último Informe de "EduTrend" realizado por el Observatorio del Tecnológico de Monterrey (Tecnológico de Monterrey, 2015), que la sitúa como una tecnología con un tiempo de adopción en los centros del Tecnológico de Monterrey de entre uno a dos años. La RA, en concordancia con Cabero y García (2016) y Barroso, et al. (2016), es una tecnología que permite la combinación de información digital e información física en tiempo real por medio de distintos soportes tecnológicos como, por ejemplo, las "tablets" o los "smartphones", para generar un nuevo escenario formativo enriquecido. Por su parte, Fombona et al., (2012) y Neven et al. (2011) definen la RA como una tecnología que permite ampliar las imágenes de la realidad, a partir de su captura por la cámara de un equipo informático o dispositivo móvil avanzado que añade elementos virtuales para la elaboración de una realidad mixta con objetos informáticos.

Por su parte, Cabero y García (2016), señalan sus propiedades más significativas: ser una realidad mixta, integrada en tiempo real, que posee una diversidad de capas de información digital, que es interactiva y que, mediante su utilización, enriquecemos o alteramos la información. La RA ofrece numerosas posibilidades educativas y un inmenso potencial para mejorar el aprendizaje y la enseñanza (Bacca et al., 2014; Prendes, 2015). Además, proporciona a los usuarios el acceso a un contenido multimedia rico, variado y significativo, facilitándoles un contexto relevante y con el que poder interactuar de manera inmediata (Cabero y García, 2016). Como señalan Di Serio et al. (2013) los sistemas de RA se caracterizan por tres propiedades básicas: a) combinar objetos reales y virtuales en un entorno real, b) alineación de objetos reales y virtuales entre sí, y c) ejecutarlos de forma interactiva y en tiempo real. Desde un punto de vista tecnológico, Cabero y Barroso (2016) aunando las propuestas de diferentes autores nos señalan los diferentes recursos y dispositivos tecnológicos que se necesitan para la producción y observación de objetos en RA, en concreto los autores nos señalan los siguientes:

“1) Un elemento que capture la imagen de la realidad que están viendo los usuarios (pantalla del ordenador, un teléfono, o una videoconsola); 2) Un dispositivo donde proyectar la mezcla de las imágenes reales con las imágenes sintetizadas (pueden servir los tres citados anteriormente); 3) Un elemento de procesamiento o varios que trabajen conjuntamente cuya función es la de interpretar la información del mundo real que recibe el usuario, generar la información virtual que cada servicio concreto necesite y mezclarla de forma adecuada (ordenadores, móviles o videoconsolas); 4) Un tipo de software específico para la producción del programa; 5) Un activador de la realidad aumentada o marcadores que pueden ser códigos $Q R$, objetos físicos, GPS...); y 6) Un servidor de contenidos donde se ubica la información virtual que queremos incorporar a la realidad". La RA precisa de estudios contextualizados en los procesos formativos que permitan identificar sus ventajas y limitaciones; una tecnología cuya funcionalidad y practicidad viene siendo puesta de relieve por diferentes investigadores (Prendes, 2015; Garay et al., 2017).

\section{ESCENARIO DEL ESTUDIO}

La experiencia innovadora inmersiva se desarrolla a través de un Seminario de Tecnologías Emergentes 
implementado durante los meses de enero y febrero del curso académico 2016-17 en dos asignaturas: "Tecnologías de la Información y la Comunicación en Educación Social", correspondientes ambas al primer año de la titulación del Grado de Educación Social y Doble Grado de Educación Social y Trabajo Social de la Facultad de Ciencias Sociales de la Universidad Pablo de Olavide, de Sevilla (España) con una carga de 7,3 Créditos ECTS (European Credit Transfer System). Con respecto al programa de estudios, la asignatura de "Tecnologías de la Información y la Comunicación en Educación Social”, pertenece al área de Didáctica y Organización Educativa y se articula alrededor de diversos bloques temáticos; en nuestro caso, corresponde al cuarto núcleo de contenidos denominado: "Temas sociales/transversales", en concreto al Tema 9: "La Realidad Aumentada en escenarios formativos sociales". El organigrama conceptual de la asignatura se puede visualizar en el siguiente enlace: http://cort.as/x-Ez. La acción formativa perseguía los siguientes objetivos: 1) Conocer las nuevas aplicaciones de RA en escenarios formativos y sus posibilidades pedagógicas en contextos educativos. 2) Generar en el estudiantado actitudes proactivas ante las tecnologías aumentadas y 3) Desarrollar en el alumnado competencias de uso didáctico de las aplicaciones de RA presentada en el seminario formativo. La actividad se desarrolló durante tres sesiones de dos horas de duración donde el alumnado de ambas titulaciones experimentaba contenidos y competencias de las asignaturas implicadas por medio de aplicaciones basadas en la RA y analizaban las posibilidades y limitaciones de la RA en escenarios formativos. Asimismo, se acompañaba la parte teórica con el uso práctico por parte del estudiantado de diversas aplicaciones de RA orientadas al contexto educativo, tales como: Quiver, ZOOKAZAM, CHROMVILLE, Anatomy 4D, ArFlashcards. Para ello, se utilizaban diferentes "tablets", marcadores impresos en papel (proporcionados por el docente) y los teléfonos móviles de los estudiantes que descargaban las apps de las aplicaciones para el uso de la RA. Asimismo, en el edublog de la asignatura se creó una pestaña denominada: "Realidad Aumentada y Virtual" que recopilaba numerosas app de carácter educativo, tutoriales y noticias de interés (http://cort.as/x-F4). (Figuras 1 y 2 ).
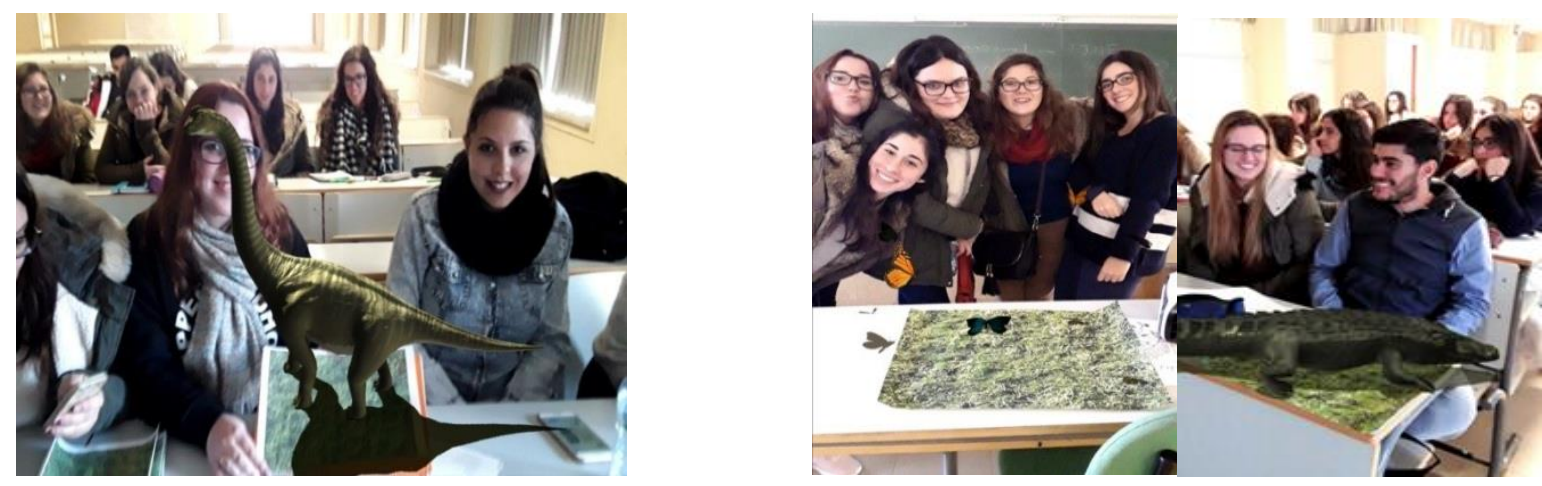

Fig. 1: Aplicaciones con RA y estudiantes de la Universidad Pablo de Olavide 2016-17 (Sevilla, España).

Al finalizar el seminario formativo se solicitaba a cada estudiante cumplimentar un cuestionario elaborado ad hoc titulado: "Uso didáctico de la RA", para indagar qué aplicación utilizadas en la actividad basada en RA era la más motivadora, analizarán los ámbitos preferentes de intervención social para utilizar estas tecnologías inmersivas y expusieran las ventajas y limitaciones de la RA en el contexto educativo.
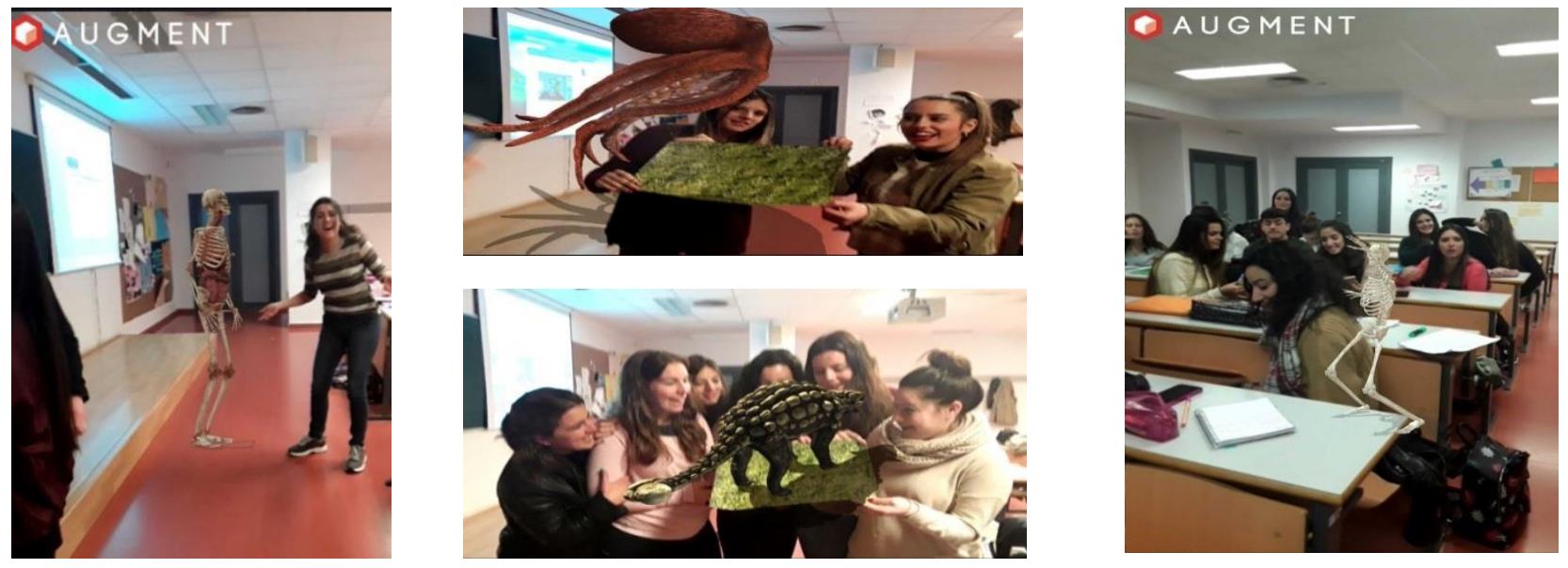

Fig. 2: Aplicaciones con RA y estudiantes de la Universidad Pablo de Olavide 2016-17 (Sevilla, España).

En última instancia, cada estudiante tenía que elaborar un edublog personal para la asignatura sobre las evidencias de las actividades realizadas en la asignatura, siendo unas de sus pestañas la Realidad 
Aumentada donde debían de responder a estas tres cuestiones: 1) ¿Qué es la Realidad Aumentada (RA) (Augmented Reality)? Ventajas e Inconvenientes de la RA en los escenarios formativos. 2) Realizar un breve comentario (máx. 300 palabras) sobre los ámbitos preferentes de intervención social (Infancia, personas adultas, tercera edad, atención comunitaria...) que se pueden aplicar con esta tecnología emergente (RA) y 3) ¿Qué aplicación de Realidad Aumentada vistas en el Seminario de Tecnologías Emergentes (Zookazam, Anatomy 4D, Quiver, Chromville, Ar Flashcards, etc. te ha gustado más? ¿Por qué? En la Figura 3, se visualizan dos ejemplos correspondientes a estudiantes de la asignatura.

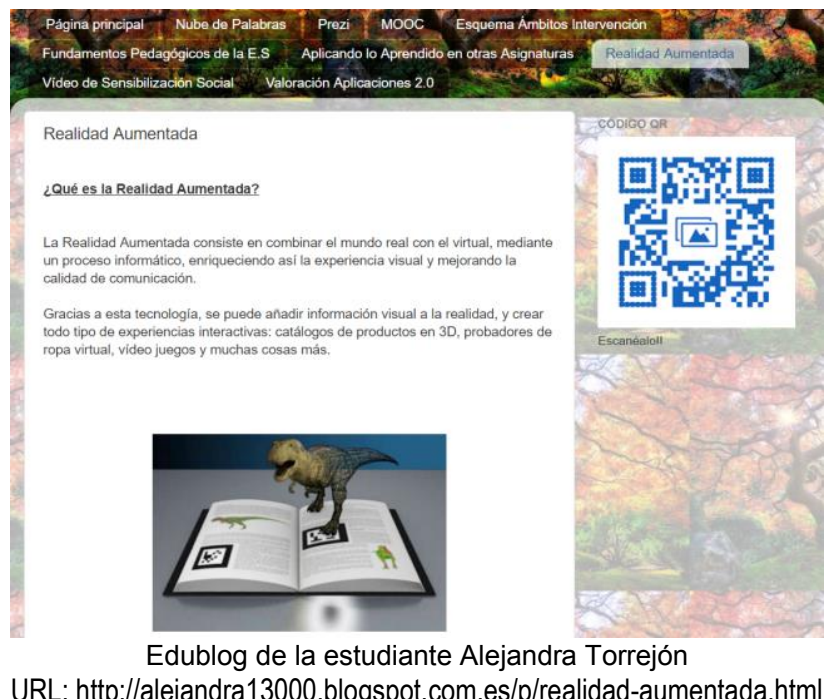

URL: http://alejandra13000.blogspot.com.es/p/realidad-aumentada.html
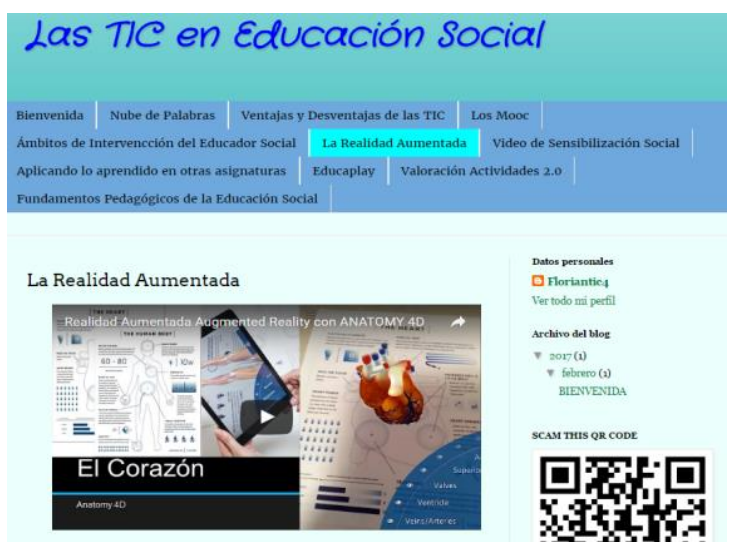

La realidad aumentada. Ventajas e Inconvenientes. La realidad aumentada hace referencie a la visualización directa o indirecta de
elementos del mundo real combinados con elementos virtuales generados por un

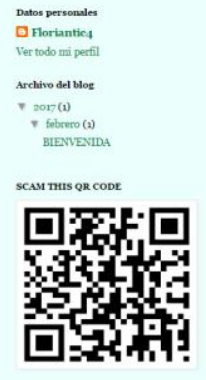

Edublog de la estudiante Florián Chacón URL: http://floriantic4.blogspot.com.es/p/la-realidad-aumentada.html

Fig. 3: Edublogs de estudiantes de la titulación de Grado de Educación Social sobre la aplicación de la RA.

\section{METODOLOGÍA}

Abordamos un estudio exploratorio cuyo principal objetivo es conocer las posibilidades educativas de la RA en la educación universitaria. Para ello, partimos de una concepción metodológica basada en la investigación-acción colaborativa virtual por parte del estudiante universitario como una manera de fomentar sus propias competencias genéricas y específicas en el Espacio Europeo de Educación Superior (PoolCibrian y Martínez-Guerrero, 2013). Esta concepción metodológica se fundamenta en la interdependencia positiva que fomenta la responsabilidad grupal del trabajo colaborativo. Las experiencias aportadas por Millis y Rhem (2010), evidencian que, al emplear el trabajo colaborativo como una estrategia en el aula universitaria, se contribuye a potenciar el aprendizaje, al permitir la confrontación de puntos de vista y opiniones, ayuda a revalorizar la perspectiva propia y facilita el intercambio con el otro, pues activa y conduce al aprendizaje para abordar con éxito situaciones comunicativas entre iguales. Los enfoques cuantitativos no son capaces de describir capacidades cognitivas en entornos de aprendizaje virtuales. Las técnicas estadísticas usuales conllevan una concepción categorial y distributiva de las estructuras, lo que ocasiona que sus resultados sean siempre distribuciones (uni o multivariadas) de atributos individuales. Por lo tanto, optamos por una metodología con un enfoque cualitativo que nos permite analizar con mayor profundidad la funcionalidad, limitaciones y posibilidades del uso didáctico de la RA por estudiantes que han empleado la misma en procesos formativos.

El análisis cualitativo se ha fundamentado en un proceso de codificación y categorización estructurado en dos etapas: la etapa descriptiva y la etapa interpretativa. El procedimiento se organizó en tres fases: 1) Fase 1: "Segmentación e identificación de unidades de significado y agrupación en categorías descriptivas". 2) Fase 2: "Construcción de un sistema de núcleos temáticos emergentes o metacategorías" y 3) Fase 3: "Identificación de dominios cualitativos (análisis secuencial y transversal de las metacategorías)". La muestra participante estuvo compuesta por 117 estudiantes de la Universidad Pablo de Olavide con las siguientes características: 60 estudiantes (8 hombres y 58 mujeres), correspondientes al primer curso del Doble Grado en Trabajo Social y Educación Social (Asignatura: "TIC y Educación Social”) y 57 estudiantes (6 hombres y 51 mujeres), correspondientes al primer curso del Grado de Educación Social (Asignatura: "TIC y Educación Social"). Se diseñó el cuestionario "Uso didáctico de la RA" (Su enlace es: https://goo.gl/forms/STik3sl9KdPzZi773).

El diseño del cuestionario partió de las consideraciones teóricas realizadas por Barroso y Gallego-Pérez (2017) y se revisó por juicio de expertos para poder delimitar las principales posibilidades educativas de la RA aplicada en contextos formativos de la Educación Superior. El objetivo principal del cuestionario era recabar la opinión de los estudiantes conforme a aspectos descriptivos valorativos de las diferentes 
herramientas propuestas y, principalmente, sobre tres ámbitos de opinión: 1) “¿Cuáles son los ámbitos preferentes de intervención social (Infancia, personas adultas, tercera edad, atención comunitaria...) que se pueden aplicar con esta tecnología emergente (RA)"? 2) “¿Cuáles son las ventajas de la RA aplicadas a la formación?” y 3) “¿Cuáles son las limitaciones de la RA aplicadas a la formación?"

\section{RESULTADOS Y DISCUSIÓN}

El proceso de reducción de datos a macrocategorías, categorías y unidades de significado fue un proceso complejo en el que se codificaron mediante el programa Atlas-Ti (versión 7.0), 418 unidades de significado textuales agrupadas en 3 dominios-dimensiones y 15 categorías deductivo-inductivas. Después de un proceso de comparación intercategorías hemos identificado y definido las siguientes unidades correspondientes a las tres dimensiones de la investigación: 1) "ámbitos preferentes de intervención social con RA" (3 categorías); 2) "ventajas de la RA aplicadas a la formación" (7 categorías) y 3) "limitaciones de la RA aplicadas a la formación" (5 categorías) en torno a los cuales se han agrupado todas las unidades de significado.

La primera dimensión analizada es la percepción de la funcionalidad de la RA sobre los principales ámbitos de intervención asociados a las competencias profesionales de los dos grados universitarios (Tabla 1).

Tabla 1: Dimensión 1: "ámbitos preferentes de intervención social con RA".

\begin{tabular}{|c|c|c|c|c|}
\hline $\begin{array}{l}\text { Dimensión 1: ámbitos preferentes de } \\
\text { intervención social con } R A\end{array}$ & Casos/Citas & $\%$ Casos & Nb Words & $\%$ Words \\
\hline Infancia. & 51 & $47,6 \%$ & 97 & $51,3 \%$ \\
\hline Tercera Edad. & 32 & $32,4 \%$ & 65 & $37,9 \%$ \\
\hline Adolescentes. & 21 & $20,0 \%$ & 23 & $10,8 \%$ \\
\hline Total & 104 & $100 \%$ & 185 & $100 \%$ \\
\hline
\end{tabular}

Los estudiantes han considerado mayoritariamente que la RA es aplicable en todas las etapas evolutivas y formativas del ser humano, principalmente en la infancia $(N=95 / 51,3 \%)$ y en la tercera edad $(N=65 / 37,9 \%)$ debido a sus condiciones de adaptabilidad en cualquier contexto que se presente. Si nos basamos en el ámbito educativo, la RA es una gran aliada para crear una interconexión entre lo virtual y la realidad y ayuda al estudiante a reforzar el aprendizaje y acercarse más a los conocimientos. Además, al ser una dinámica diferente a los que los alumnos suelen estar acostumbrados, hace que estos muestren más atención consiguiendo así ampliar sus conocimientos. Este incremento de la atención también ha sido documentado en otros estudios de RA (Cuendet, et al, 2013; Tsai, et al., 2013). De igual manera, se puede utilizar fuera del ámbito educativo en personas adultas y de tercera edad para tratar diferentes situaciones psicosociales.

Asimismo, los estudiantes han considerado que sería interesante su uso en diferentes contextos formativos como un recurso que promueve la creatividad, la imaginación y la curiosidad de la persona, además de construir nuevos conocimientos e interaccionar de una manera tan directa con lo virtual. Estas valoraciones se han confirmado también parcialmente en estos estudios universitarios con la aplicación del portafolio y los edublogs para mejorar la formación del alumnado en la adquisición de competencias para la atención de grupos sociales en desventaja por edad o situación sociolaboral con resultados muy positivos (Vázquez-Cano, et al., 2015; 2016). Asimismo, también confirma los resultados de otros estudios (Di Serio, et al., 2013) en relación al incremento de la atención y la motivación del alumnado en las actividades de enseñanzaaprendizaje. Por lo tanto, la realidad aumentada puede ayudar a incrementar el valor de otras tecnologías en el aspecto formativo y práctico del desarrollo de las competencias profesionales y académicas del alumnado universitario. Las relaciones más profundas que se establecen entre estas posibilidades de aplicación al ejercicio profesional del educador y trabajador social se pueden visibilizar en la Figura 4.

Como se puede apreciar en la red de conceptos y categorías generada por el tratamiento cualitativo con Atlas$\mathrm{Ti}$ de las opiniones de los estudiantes, las cuatro principales áreas de intervención con RA se centran en: drogodependencia aplicada a la educación y prevención entre adolescentes, la discapacidad asociada a la demencia en personas mayores y la faceta educativa en la infancia y la educación de menores (adolescentes) en la enseñanza formal y no formal. Los estudiantes consideran que la aplicación de estrategias didácticas y prácticas basadas en la RA en estos ámbitos podrían mejorar la adquisición de competencias por parte de los profesionales y, asimismo, servir de apoyo y mejora de las condiciones y tratamiento de los aspectos reseñados en los diferentes colectivos sociales. La segunda dimensión analizada es la percepción de la funcionalidad de la RA sobre las ventajas de la RA aplicadas a la formación (Tabla 2). 


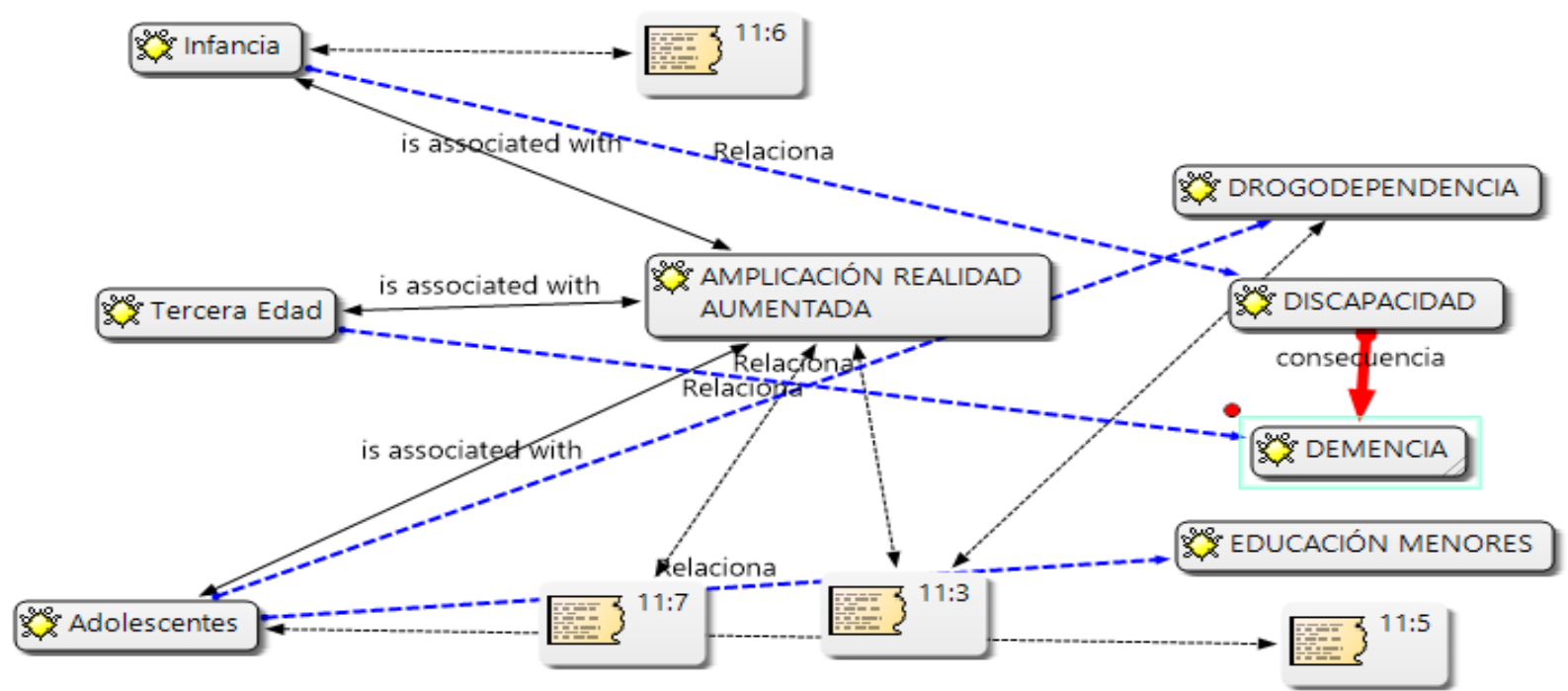

Fig. 4: Red Atlas-Ti. Ámbitos preferentes de intervención social con RA.

Tabla 2: Dimensión 2: "ventajas de la RA aplicadas a la formación”.

\begin{tabular}{|c|c|c|c|c|}
\hline Dimensión 2: ventajas de la $R A$ aplicadas a la formación & Casos/Citas & $\%$ Casos & Nb Words & $\%$ Words \\
\hline Activación procesos cognitivos. & 24 & $15,20 \%$ & 62 & $14,70 \%$ \\
\hline Formación de actitudes de reflexión. & 26 & $17,10 \%$ & 69 & $16,90 \%$ \\
\hline $\begin{array}{l}\text { Desarrollo de habilidades cognitivas, espaciales, perceptivo } \\
\text { motoras y temporales. }\end{array}$ & 19 & $12,20 \%$ & 43 & $11,90 \%$ \\
\hline Estimulación de la atención, concentración, y memoria inmediata. & 21 & $13,10 \%$ & 55 & $12,00 \%$ \\
\hline $\begin{array}{l}\text { Ofrece un entorno amigable de comunicación para el trabajo } \\
\text { educativo. }\end{array}$ & 16 & $11,30 \%$ & 34 & $12,80 \%$ \\
\hline Aumenta la actitud positiva de los estudiantes. & 25 & $16,10 \%$ & 61 & $15,90 \%$ \\
\hline Potencia escenarios formativos más motivadores. & 24 & $15,00 \%$ & 59 & $15,80 \%$ \\
\hline Total & 155 & $100 \%$ & 383 & $100 \%$ \\
\hline
\end{tabular}

Como se puede observar en el análisis de frecuencias de citas y palabras, la RA aplicada al ámbito formativo permite actividad los procesos cognitivos ( $N=62 / 14,7 \%)$, generando nuevas ideas, conocimientos u opiniones acerca del mundo que rodea al estudiante. Da lugar a la reflexión, a la explicación de fenómenos y aporta soluciones a determinados problemas ( $\mathrm{N}=69 / 16,9 \%)$. Asimismo, permite desarrollar habilidades cognitivas, espaciales, perceptivo motoras y temporales en los estudiantes $(N=43 / 11,9 \%)$. Estos resultados también fueron puestos de relieve en otros estudios que tomaban como referencia la simulación de habilidades en contextos de emergencia o accidentes (Tsai, et al., 2013). Determinadas aplicaciones de la RA son consideradas por los estudiantes como estimuladoras de la atención, concentración, memoria inmediata, memoria mediata y del razonamiento ( $N=55 / 12,0 \%$ ). Estos resultados también han sido refrendados por Nadolny (2017) con experiencias educativas que enriquecen los tradicionales medios impresos con recursos de realidad aumentada. Su empleo, posibilita un entorno amigable de comunicación para el trabajo educativo y ayuda a la comprensión del objeto de estudio ( $\mathrm{N}=$ $34 / 12,8 \%)$. Aumenta la actitud positiva de los estudiantes ante el aprendizaje, así como su motivación, reforzando capacidades y competencias $(\mathrm{N}=61 / 15,9 \%)$.

Los estudiantes de ambos grados consideran que se potencia el uso de la RA, escenarios formativos más motivadores, colaborativos e interactivos y ayudan a una educación más abierta ( $N=59 / 15,8 \%)$. Resultados similares han sido mostrados en la formación de los futuros docentes de educación primaria (MorenoMartínez y Leiva-Olivencia, 2017). La motivación que produce la RA en los procesos formativos también se ha puesto de relieve en diferentes estudios (Lin et al., 2013; Leiva y Moreno, 2015; Cózar et al., 2015); si bien en ambos estudios, se considera que el estudiante no debe centrarse el manejo de los aparatos, sino que debe contemplar la funcionalidad didáctica que subyace detrás de la tecnología para poder aplicarla correctamente tanto en el mundo académico como en el profesional. Las relaciones entre las ventajas de la RA al ámbito formativo se pueden visualizar en la red de Atlas-Ti de la Figura 5. 


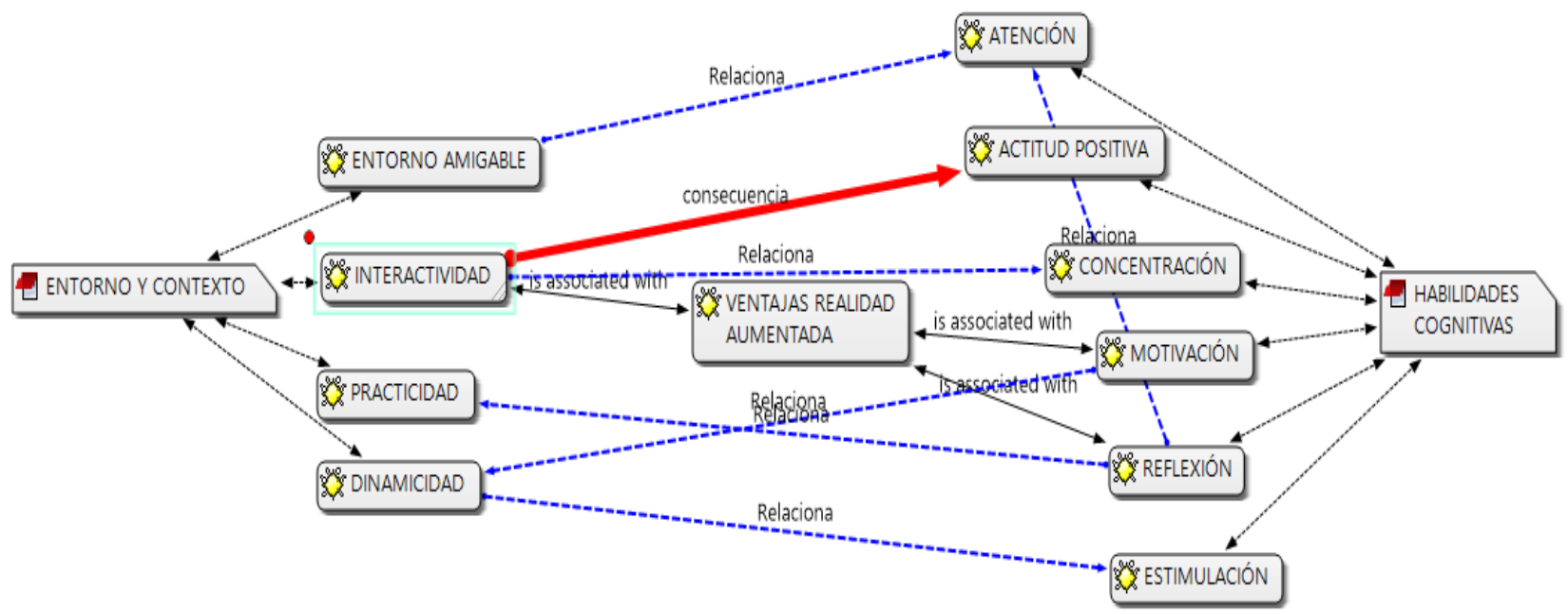

Fig. 5: Red Atlas-Ti. Ventajas de la RA aplicadas a la formación del estudiante universitario de Educación y Trabajo social.

La red de análisis cualitativo nos muestra que las ventajas de la realidad aumentada se ven condicionadas desde dos parámetros interpretativos: el "entorno y el contexto" y las "habilidades cognitivas". Los estudiantes consideran que la potencialidad de esta tecnología para el desarrollo de habilidades cognitivas en los usuarios parte de una adecuada selección del entorno y escenario en el que se van a aplicar. Por lo tanto, su adecuación a la situación y aspecto a estudiar o mejorar está en la base de su éxito, no es adecuada su utilización en cualquier contexto o entorno. Esta necesidad de una aplicación al entorno y contexto más adecuado, principalmente en el desarrollo de actividades en la enseñanza secundaria superior, también ha sido destacada por Wojciechowski y Cellary (2013) en estudios de Química y por Nielsen, et al. (2016) en estudios de ciencias. La tercera dimensión analizada es la percepción de las limitaciones de la RA aplicadas a la formación (Tabla 3).

Tabla 3: Dimensión 3: "las limitaciones de la RA aplicadas a la formación”.

\begin{tabular}{|c|c|c|c|c|}
\hline $\begin{array}{c}\text { Dimensión 3: limitaciones de la RA aplicadas a la } \\
\text { formación }\end{array}$ & $\begin{array}{l}\text { Casos/ } \\
\text { Citas }\end{array}$ & $\%$ Casos & Nb Words & $\%$ Words \\
\hline Elevado coste económico. & 35 & $24,70 \%$ & 91 & $25,00 \%$ \\
\hline Puede incrementar la brecha digital interactiva & 28 & $18,00 \%$ & 78 & $19,00 \%$ \\
\hline Requiere mayor formación en los educadores. & 37 & $26,30 \%$ & 98 & $27,50 \%$ \\
\hline Incrementa una sociabilidad virtual menos humana. & 31 & $18,00 \%$ & 81 & $15,00 \%$ \\
\hline $\begin{array}{l}\text { Baja adaptabilidad a las necesidades especiales de } \\
\text { los estudiantes }\end{array}$ & 28 & $13,00 \%$ & 47 & $13,50 \%$ \\
\hline Total & 159 & $100 \%$ & 395 & $100 \%$ \\
\hline
\end{tabular}

Podemos observar en el cómputo de frecuencias que, para realizar una adecuada implementación de la RA, existen también una serie de inconvenientes. Entre ellos, destaca el requerimiento de inversión económica que garantice el acceso a esta tecnología en las aulas y centros formativos ( $N=91 / 25,0 \%)$. Esta situación puede provocar que se acreciente la brecha digital entre colectivos menos favorecidos socialmente $(N=78 / 19,0 \%)$. Asimismo, también se requiere una formación específica para manipular la tecnología y poder proponer actividades formativas $(\mathrm{N}=98 / 27,5 \%)$. Sin esta adecuada formación, su uso puede llegar a resultar contraproducente porque podría generar un retraso en el desarrollo de competencias y contenidos en la programación de la enseñanza. Su uso, también se asocia con un incremento del distanciamiento humano, lo que puede terminar acrecentando una "didáctica fría", poco asertiva que deshumanice los procesos de enseñanza-aprendizaje $(\mathrm{N}=81 / 15,0 \%)$. $\mathrm{Y}$, por último, se reseña que muchos de estos sistemas no se adaptan a las diferentes necesidades especiales que puedan tener los estudiantes en las aulas ( $N=47 / 13,5 \%$ ).

La literatura científica también ha evidenciado que la RA puede provocar problemas en su aplicación en procesos educativos principalmente por la dificultad que entraña la didáctica "deshumanizada" (Chiang, et., 2014). Las relaciones entre las limitaciones de la RA al ámbito formativo se pueden visualizar en la red de Atlas-Ti de la Figura 6. La red de relaciones conceptuales nos muestra que las limitaciones de la RA a los procesos formativos también se relacionan entre ellas. Así el menor contacto humano "deshumanización" pueden incrementar la brecha digital y, a su vez, la brecha digital puede dificultar la formación y la adquisición de esta tecnología en las aulas. 


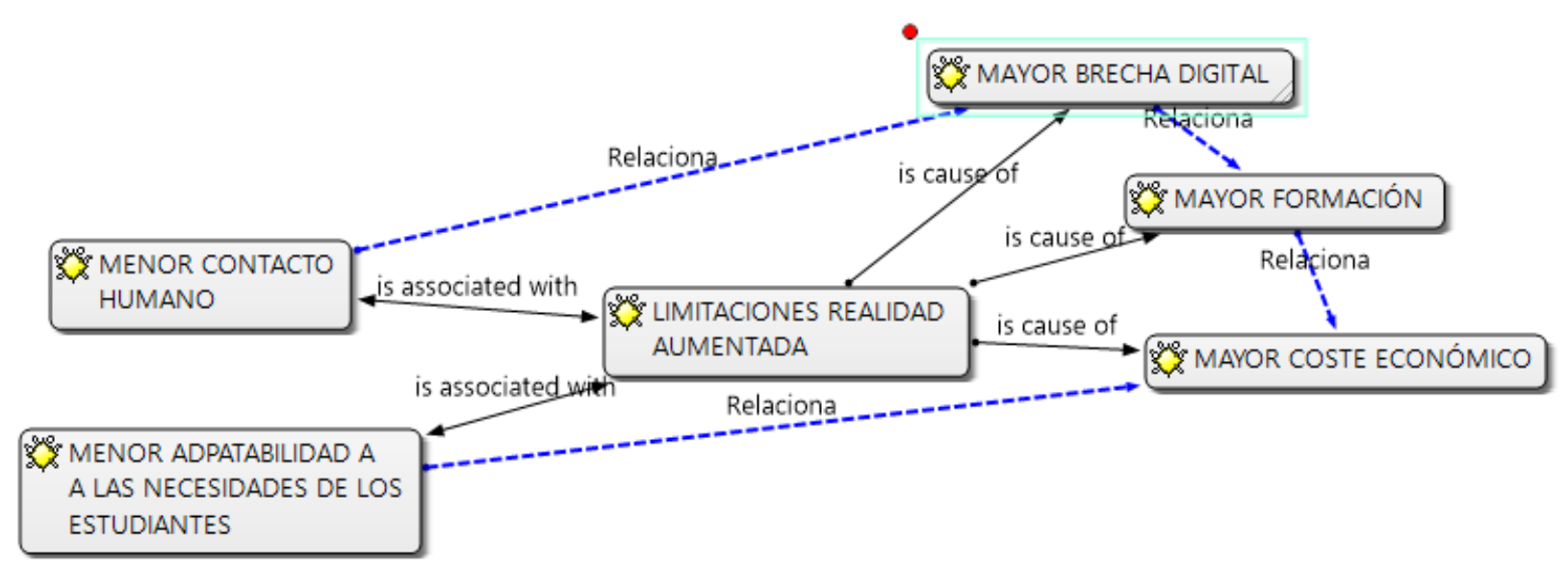

Fig. 6: Red Atlas-Ti. Limitaciones de la RA aplicadas a la formación del estudiante universitario de Educación y Trabajo social.

\section{CONCLUSIONES}

El análisis de la experiencia didáctica de aplicación de la RA con alumnado universitario nos permite aseverar como primera conclusión que la utilización de objetos con base en la tecnología aumentada en la Educación Superior, despierta verdadero interés entre los estudiantes. Este extremo, también ha sido corroborado por diferentes estudios internacionales que muestran alto niveles de satisfacción cuando el alumnado utiliza esta tecnología y el aumento significativo de los niveles de motivación cuando los estudiantes están inmersos en acciones formativas con RA. Junto la motivación, se ha evidenciado que el uso de la RA potencia escenarios formativos más motivadores, colaborativos e interactivos y ayuda a una educación más abierta y creativa.

Asimismo, la percepción del alumnado de Educación Superior en las áreas de Ciencias Sociales de este estudio ha evidenciado en sus apreciaciones que el empleo de actividades con base en la RA posibilita una mayor reflexión e incrementa la actitud positiva y los procesos cognitivos aplicados a los contenidos de las titulaciones en Educación y Trabajo Social. Por otra parte, como también se infiere del análisis de los resultados por parte de los estudiantes, resulta interesante ver cómo la RA es aplicable no solo en el proceso de enseñanza-aprendizaje, sino como un recurso útil y con gran potencial en el desempeño de sus competencias profesionales como Educadores y Trabajadores Sociales. Así, con respecto a su aplicación en el ámbito profesional del Educador y Trabajador social, los estudiantes participantes han reseñado que la RA puede resultar de especial interés para el tratamiento de problemas de drogodependencia y su prevención entre adolescentes, la discapacidad asociada a la demencia en personas mayores y la faceta educativa en la infancia y la educación de menores (adolescentes) en la enseñanza formal y no formal. La aplicación de esta tecnología en el tratamiento psicosocial de estos problemas podría mejorar la adquisición de competencias por parte de los profesionales y, asimismo, servir de apoyo y mejora de las condiciones y tratamiento de los aspectos reseñados en los diferentes colectivos sociales.

Además, el empleo de la RA ha sido útil en la construcción de competencias emergentes sobre utilización de TIC, capacidad de trabajo en equipo, el descubrimiento de nuevos recursos didácticos inmersivos útiles en los escenarios de la Educación Social y el Trabajo Social, desconocidos por la mayoría del estudiantado, lo cual puede ayudar al desarrollo de nuevos procesos formativos desde una perspectiva indagadora, constructivista y ubicua. En este sentido, queremos destacar que las actividades didácticas con base en la RA pueden ser especialmente útiles en estudios preuniversitarios y de enseñanza superior relacionados con las áreas de Humanidades y Ciencias Sociales ya que permite un acceso a contenidos, muchas veces presentados de forma más unidireccional mediante medios impresos que no incrementan la motivación o requieren un mayor esfuerzo de abstracción entre los estudiantes. Con este recurso, los contenidos se visualizan de una forma más creativa, dinámica y real consiguiendo una didáctica más atractiva, innovadora y motivadora en cualquier nivel educativo.

A pesar de estos supuestos beneficios, creemos que se debe ahondar en las propuestas y modelos de integración de la RA teniendo en cuenta que su desarrollo debe contextualizarse debidamente; tomando en consideración los temas y recursos disponibles y cuál es la formación del profesorado; ya que sin la adecuada formación, su uso puede llegar a resultar contraproducente pudiendo generar un retraso en el desarrollo de competencias y contenidos en la programación de la enseñanza. Además, hay que tener muy presentes los recursos con los que cuentan las instituciones educativas y los propios estudiantes para no generar una mayor brecha digital o discriminación por motivos económicos o de acceso a la tecnología. Asimismo, hay que garantizar que esta tecnología incorpore mecanismos de usabilidad y adaptabilidad a las diferentes necesidades especiales que puedan tener los estudiantes en las aulas. 
Por último, creemos necesario no obviar estas tecnologías y su uso en las aulas; ya que además de servir como nuevos medios para vehiculizar el aprendizaje, suponen la adquisición de competencias necesarias entre los estudiantes para su correcto y adecuado futuro profesional. En este camino, las universidades deben jugar un nuevo papel como propulsoras de competencias que el futuro egresado deberá manejar en su desenvolvimiento académico, personal y profesional a lo largo de la vida.

\section{AGRADECIMIENTOS}

El presente trabajo se inserta en el proyecto de investigación denominado "Realidad Aumentada para Aumentar la Formación". Diseño, Producción y Evaluación de Programas de Realidad Aumentada para la Formación Universitaria (RAFODIUN) (EDU2014-57446-P). También, se agradece a los estudiantes de las asignaturas de Tecnologías de la Información y la Comunicación en Educación Social, por el apoyo en la realización de la experiencia innovadora.

\section{REFERENCIAS}

Álvarez-Marín, A., M. Castillo-Vergara, J. Pizarro-Guerrero y E. Espinoza-Vera, Realidad Aumentada como Apoyo a la Formación de Ingenieros Industriales, Formación Universitaria, 10 (2), 31-42 (2017)

Bacca, J., S. Baldiris, R. Fabregat, S. Graf y J. Kinshuk, Augmented Reality Trends in Education: A Systematic Review of Research and Applications, Educational Technology \& Society, 17 (4), 133-149 (2014)

Barroso, J., J. Cabero y A.M. Moreno-Fernández, La utilización de objetos de aprendizaje en realidad aumentada en la enseñanza de la medicina, International Journal of Technology and Educational Innovation 2 (2), 77-83 (2016)

Barroso, J. y O.M. Gallego-Pérez, Producción de recursos de aprendizaje apoyados en Realidad Aumentada por parte de estudiantes de magisterio, Revista de Educación Mediática y TIC (Edmetic), 6(1), 23-38 (2017)

Bressler, D. M. y A. M. Bodzin, A mixed methods assessment of students' flow experiences during a mobile augmented reality science game, Journal of Computer Assisted Learning, 29 (6), 505-517 (2013)

Cabero, J. y J. Barroso, Posibilidades educativas de la realidad aumentada, New Approaches in Educational Research, 5 (1), 46-52 (2016)

Cabero, J. y F. García (Coord.), Realidad aumentada. Tecnología para la formación, Síntesis, Madrid, España (2016)

Chang, H., K. Wu y Y. Hsu, Integrating a mobile augmented reality activity to contextualize student learning of a socioscientific issue, British Journal of Educational Technology, 44, 3, 95-99 (2013)

Chiang, T.-H.-C., S.-J.-H. Yang y G.-J. Hwang, An Augmented Reality-based Mobile Learning System to Improve Students' Learning Achievements and Motivations in Natural Science Inquiry Activities, Educational Technology \& Society, 17 (4), 352-365 (2014)

Chin-Ming, C. y T. Yen Nung, Interactive augmented reality system for enhancing library instruction in elementary schools, Computers \& Education, 59, 638-652 (2011)

Cózar, R., M. De Moya, J. Hernández y J. Hernández, Tecnologías emergentes para la enseñanza de las Ciencias Sociales. Una experiencia con el uso de Realidad Aumentada en la formación inicial de maestros, Digital Education Review, 27, 138-153 (2015)

Cuendet, S., Q. Bonnard, S. Do-Lenh y P. Dillenbourg, Designing augmented reality for the classroom, Computers \& Education, 68, 557-569 (2013)

Di Serio, A., M.B. Ibáñez y C. Delgado, Impact of an augmented reality system on students' motivation for a visual art course, Computers \& Education, 68, 586-596 (2013)

Fombona, J., M.A. Pascual y M.F. Madeira, M.F, Realidad Aumentada, una evolución de las aplicaciones de los dispositivos móviles. Píxel-Bit, Revista de Medios y Educación, 41, 197-210 (2012)

Furth, B. Handbook of Augmented Reality, Springer, Florida, USA (2011)

Garay, U., E. Tejada y I. Maíz, Valoración de objetos educativos enriquecidos con realidad aumentada: una experiencia con alumnado de máster universitario. Píxel-Bit, Revista de Medios y Educación, 50, 19-31 (2017) 
Johnson, L., S. Adams-Becker, M. Cummins, V. Estrada, A. Freeman y H. Ludgate, Technology Outlook for Australian Tertiary Education 2013-2018: An NMC Horizon Project Regional Analysis, The New Media Consortium, Austin, Texas, USA (2013)

Kim, K., J. Hwang y H. Zo, Understanding users' continuance intention toward smartphone augmented reality applications, Information Development, 32 (2), 161-174 (2016)

Kipper, G. y J. Rampolla, Augmented reality, Amsteda, Syngress, Holland (2012)

Leiva, J.J. y N. Moreno, Tecnologías de geolocalización y realidad aumentada en contextos educativos: experiencias y herramientas didácticas, Revista Didáctica, Innovación y Multimedia (DIM), (en línea) https://goo.gl/zzc3Rq, 31 (2015)

Lin, T., H. Been-Lirn, N. Li, H. Wang y Ch. Tsai, An investigation of learners' collaborative knowledge construction performances and behavior patterns in an augmented reality simulation system, Computers \& Education, 68, 314-321 (2013)

Moreno-Martínez, N. y J.J. Leiva-Olivencia, Experiencias formativas de uso didáctico de la realidad aumentada con alumnado del grado de educación primaria en la universidad de Málaga, Revista de Educación Mediática y TIC (Edmetic), 6 (1), 81-104 (2017)

Millis, B.J. y J. Rhem, Cooperative Learning in Higher Education. Across the Disciplines, across the Academy, Stylus Publishing, Virginia, USA (2010)

Nadolny, L., Interactive print: The design of cognitive tasks in blended augmented reality and print documents, British Journal of Educational Technology, 48, 814-823 (2017)

Neven, A. M., H. Hala y I. Mohamed, ARSC: Augmented Reality Student Card An Augmented reality Solution for the educational field, Computers \& Education, 56, 1045-1061 (2011)

Nielsen, B.L., H. Brandt y H. Swensen, Augmented Reality in science education-affordances for student learning, Nordic Studies in Science Education, 12 (2), 157-174 (2016)

Pool-Cibrian, W.J. y J.I. Martínez-Guerrero, Autoeficacia y uso de estrategias para el aprendizaje autorregulado en estudiantes universitarios, Rev. Electrónica de Investigación Educativa, 15(3), 21-37 (2013)

Prendes, C., Realidad aumentada y educación: análisis de experiencias prácticas, Pixel-Bit, Revista de Medios y Educación, 46, 187-203 (2015)

Sevillano, M.a.L. y E. Vázquez-Cano, The impact of digital mobile devices in Higher Education, Educational Technology \& Society, 18 (1), 106-118 (2015)

Tecnológico de Monterrey, Reporte EduTrends. Radar de Innovación Educativa 2015, Tecnológico de Monterrey, Monterrey, México (2015)

Tsai, M. P. Liu y J. Yau, Using electronic maps and augmented realitybased training materials as escape guidelines for nuclear accidents: An explorative case study in Taiwan, British Journal of Educational Technology, 44 (1), 18-21 (2013)

Vázquez-Cano, E., E. López Meneses y J. L. Sánchez-Serrano, Analysis of social worker and educator's areas of intervention through multimedia concept maps and online discussion forums in Higher Education, Electronic Journal of e-Learning, 13 (5), 333-346 (2015)

Villalustre, L. Experiencias interactivas con realidad aumentada en las aulas, Octaedro, Barcelona (2016)

Wojciechowski, R. y W. Cellary, Evaluation of learners' attitude toward learning in ARIES augmented reality environments, Computers \& Education, 68, 570-585 (2013) 